\title{
A New Algorithm of Optimization of Continuous-Time Linear Observations for the Stationary Kalman-Bucy Filter
}

\author{
Yoshiki TAKEUCHI \\ Dept. of Information Science, Osaka Kyoiku University \\ 4-698-1 Asahigaoka, Kashiwara City, Osaka 582-8582, Japan \\ Tel. +81-729-78-3669, Fax. +81-729-78-3554 \\ e-mail: takeuti@cc.osaka-kyoiku.ac.jp
}

\begin{abstract}
In this paper, we are concerned with a problem of optimization of the linear observations which are used in the stationary Kalman-Bucy filter. Especially, we consider the optimization of the gain matrix in the observation. In the previous works of the author, the condition of optimality was already shown by introducing a symmetric matrix called SSNRM which is a quadratic function of the gain matrix weighted by the inverse of the noise covariance matrix. In this paper, a new numerical algorithm to compute the solution is proposed which is applicable to the observations with lesser dimensions than the signal. Most part of numerical computations of this algorithm consists of that of the solutions of Riccati and Lyapunov equations which are easily implemented. The value of SSNRM is updated by a simple scheme based on the condition of optimality. The results of numerical experiments are provided to show the efficiency of the algorithm.
\end{abstract}

\section{Introduction}

In this paper, we consider optimization of the gain matrix in the linear observation which is used for the stationary Kalman-Bucy filter. The criterion of the optimization is quadratic in both the estimation error and the gain matrix, and is given by a sum of the weighted variance of the estimation errors and a quadratic function of the gain matrix. In previous works of the author, the discrete-time problem was considered [7]-[13], and in [18], the following results were obtained for continuous-time systems.

(1) The optimal value of the observation gain matrix is not unique, but we can obtain one of them from the optimal value of the Symmetric Signal-to-Noise Ratio Matrix (SSNRM) which is a quadratic function of the gain matrix weighted by the inverse of the noise covariance;

(2) The original problem, with the observation gain matrix as the variable, is converted to an optimization problem with variables of a pair of matrices (one with the set of eigenvectors and the other diagonal matrix with the corresponding eigenvalues of SSNRM);

(3) The condition of optimality is given in terms of two symmetric matrices. One is a quadratic function of the estimation error covariance and linearly depends on the other matrix which is given by a solution of a Lyapunov equation. The condition is in the form that the first symmetric matrix has the same eigenvectors as SSNRM and also has the same eigenvalues as the observation error covariance matrix.

(4) The optimization problem is shown in the form with the variables:

(a) the error covariance matrix;

(b) the pair of matrices obtained in (2);

(c) the symmetric matrix obtained in (3) which is given by a solution of another Lyapunov equation; and is concerned with the equations:

(x) the Riccati equation of the error covariance with (a) and (b);

(y) the condition of optimality with (b) and (c);

(z) the Lyapunov equation with (a) and (c).

In [18], an algorithm to compute the solution of (x), (y) and (z) was also proposed without any proof of the convergency.

In this paper, we are concerned with developing a new recursive algorithm to compute the optimal value of SSNRM by solving ( $\mathrm{x}$ ) and (z) alternately and by updating (b) of SSNRM according to (y). To the important case of the observations with a lesser dimension than the signal, the new algorithm is applicable but the one proposed in [18] is not. The results of the numerical computation for illustrative examples are shown for better understanding of the result of this paper.

Mathematical symbols, in this paper, are used in the following way. $\boldsymbol{R}$ is the space of all real numbers, i.e., $\boldsymbol{R} \triangleq(-\infty, \infty)$. For positive integers $m$ and $n, \boldsymbol{R}^{n}$ and $\boldsymbol{R}^{m \times n}$ denote the spaces of $n$-dimensional vectors and $m \times n$-dimensional matrices whose components take values in $\boldsymbol{R}$. The prime denotes the transpose of a vector or a matrix and the Euclidean norm is $|\cdot|$. Thus, for $x \in \boldsymbol{R}^{n}, \quad|x|=\sqrt{x^{\prime} x} . \quad$ The identity matrix of any dimension is denoted by $I$. The components of a matrix are denoted by using subscripts. Thus, $[A]_{i j}$ is the $(i, j)$-component of $A$. In the case where no confusion may arise, we denote $[A]_{i j}$ simply by $a_{i j}$. If $A$ is a square matrix, $\operatorname{det}|A|$ and $\operatorname{tr}[A]$ respectively denote the determinant and the trace of $A$. We use $A>0$ and $A \geq 0$ to denote that $A$ is positive definite and nonnegative definite, respectively. The triplet $(\Omega, \mathscr{F}, P)$ is a complete probability space where $\Omega$ is a sample space with elementary events $\omega, \mathscr{F}$ is a $\sigma$-field of subsets of 
$\Omega$, and $P$ is a probability measure. $E\{\cdot\}$ denotes the expectation and $E\{\cdot \mid \mathscr{G}\}, \mathscr{G} \subset \mathscr{\mathscr { T }}$ the conditional expectation, given $\mathscr{G}$, with respect to $P . \sigma\{\cdot\}$ is the minimal sub- $\sigma$-field of $\mathscr{F}$ with respect to which the family of $\mathscr{\mathscr { H }}$-measurable sets or random variables $\{\cdot\}$ is measurable.

\section{Problem Formulation}

The formulation of the problem is almost the same as in the previous paper by the author [18]. However, in this paper, fundamental assumptions are rather weakened.

Let $\boldsymbol{x} \equiv\left\{x_{t}(\omega) ; 0 \leq t<\infty\right\}$ be an $n$-dimensional Gaussian stochastic process generated by the linear system:

$$
\left\{\begin{aligned}
d x_{t}(\omega) & =A x_{t}(\omega) d t+G d w_{t}(\omega) \\
x_{0}(\omega) & =x^{0}(\omega)
\end{aligned}\right.
$$

where $A \in \boldsymbol{R}^{n \times n}, G \in \boldsymbol{R}^{n \times d_{1}}, x^{0}(\omega)$ is a Gaussian random vector with mean $\hat{x}^{0}$ and covariance $Q^{0}$, and $\boldsymbol{w} \equiv$ $\left\{w_{t}(\omega) ; 0 \leq t<\infty\right\}$ is a $d_{1}$-dimensional standard Brownian motion process. Suppose that the available data concerned with $\boldsymbol{x}$ is given by the linear observations described by

$$
d y_{t}(\omega)=H x_{t}(\omega) d t+R d v_{t}(\omega)
$$

where $\boldsymbol{y} \equiv\left\{y_{t}(\omega) ; 0 \leq t<\infty\right\} \quad$ is an $m$-dimensional observation process, $H \in \boldsymbol{R}^{m \times n}, \quad R \in \boldsymbol{R}^{m \times d_{2}}$, and $\boldsymbol{v} \equiv$ $\left\{v_{t}(\omega) ; 0 \leq t<\infty\right\}$ is a $d_{2}$-dimensional standard Brownian motion process. Throughout this paper, we will assume the following two conditions.

(C-1) $G G^{\prime}>0$, and $R_{0} \triangleq R R^{\prime}>0$,

(C-2) $x^{0}(\omega), \boldsymbol{w}$ and $\boldsymbol{v}$ are mutually independent.

It is well-known that the least-squares estimate $\hat{x}_{t}(\omega) \triangleq$ $E\left\{x_{t}(\omega) \mid \mathscr{Y}_{t}\right\} \quad$ of $\quad x_{t}(\omega)$ based on $\mathscr{Y}_{t} \triangleq \sigma\left\{y_{s}(\omega)\right.$; $0 \leq s \leq t\}$ is given by the Kalman-Bucy filter:

$$
\left\{\begin{aligned}
d \hat{x}_{t}(\omega) & =A \hat{x}_{t}(\omega) d t+Q_{t} H^{\prime} R_{0}^{-1}\left\{d y_{t}(\omega)-H \hat{x}_{t}(\omega) d t\right\} \\
\hat{x}_{0}(\omega) & =E\left\{x^{0}(\omega)\right\},
\end{aligned}\right.
$$

and

$$
\left\{\begin{aligned}
\frac{d Q_{t}}{d t} & =A Q_{t}+Q_{t} A^{\prime}+G G^{\prime}-Q_{t} H^{\prime} R_{0}^{-1} H Q_{t} \\
Q_{0} & =E\left\{\left[x_{0}(\omega)-\hat{x}_{0}(\omega)\right]\left[x_{0}(\omega)-\hat{x}_{0}(\omega)\right]^{\prime}\right\},
\end{aligned}\right.
$$

where

$$
Q_{t} \triangleq E\left\{\left[x_{t}(\omega)-\hat{x}_{t}(\omega)\right]\left[x_{t}(\omega)-\hat{x}_{t}(\omega)\right]^{\prime}\right\},
$$

and

$$
R_{0} \triangleq R R^{\prime}
$$

In this paper, we are concerned with the stationary Kalman-Bucy filter. Namely, we assume $Q_{t} \rightarrow Q$ as $t \rightarrow \infty$, and that we have, instead of (3) and (4), the following equations:

$$
\left\{\begin{aligned}
d \hat{x}_{t}(\omega) & =A \hat{x}_{t}(\omega) d t+Q H^{\prime} R_{0}^{-1}\left\{d y_{t}(\omega)-H \hat{x}_{t}(\omega) d t\right\} \\
\hat{x}_{0}(\omega) & =E\left\{x^{0}(\omega)\right\},
\end{aligned}\right.
$$

$$
A Q+Q A^{\prime}+G G^{\prime}-Q H^{\prime} R_{0}^{-1} H Q=0 .
$$

For the optimization of performance of the least-squares state estimator (7) and (8), we introduce the following performance criterion: ${ }^{[13]}$

$$
J \triangleq \operatorname{tr}[M Q]+\operatorname{tr}\left[H N H^{\prime}\right]
$$

where $\operatorname{tr}[M Q]$ is an weighted estimation error variance, i.e.,

$$
\operatorname{tr}[M Q]=E\left\{\left[x_{t}(\omega)-\hat{x}_{t}(\omega)\right]^{\prime} M\left[x_{t}(\omega)-\hat{x}_{t}(\omega)\right]\right\},
$$

and $M \in \boldsymbol{R}^{n \times n}$ and $N \in \boldsymbol{R}^{r \times r}$ are positive-definite symmetric matrices. In (9), the second term in the right-hand side denotes the cost or the energy consumed by the observation.

Thus, we are now concerned with the following problem.

[Problem 1] Find $H \in \boldsymbol{R}^{m \times n}$ such that (9) is minimized subject to (8).

It is already shown in [18] that by a set of simple transformations of variables, Problem 1 is reduced to the special case where $N=I$. Hence, in this paper, we are only concerned with the case $N=I$. Namely, we consider

[Problem 1'] Find $H \in \boldsymbol{R}^{m \times n}$ such that

$$
J \triangleq \operatorname{tr}[M Q]+\operatorname{tr}\left[H H^{\prime}\right],
$$

is minimized subject to (8).

The key idea of the approach is an application of the orthonormal-diagonal representation of the symmetric matrix $H^{\prime} R_{0}^{-1} H$. Note that this symmetric matrix is the one which may be called a symmetric signal-to-noise ratio matrix (SSNRM). Since $H^{\prime} R_{0}^{-1} H$ is a nonnegativedefinite symmetric matrix, we can represent this matrix as the form:

$$
H^{\prime} R_{0}^{-1} H=\tilde{U} \tilde{\Xi} \tilde{U}^{\prime},
$$

where $\tilde{U}=\left[u_{1} u_{2} \cdots u_{\tilde{m}}\right] \in \boldsymbol{R}^{n \times \tilde{m}}$ is the set of eigenvectors of $H^{\prime} R_{0}^{-1} H$ corresponding to the positive eigenvalues $\xi_{i}, i=1,2, \cdots, \tilde{m}$, and

$$
\begin{gathered}
\tilde{\Xi} \triangleq \operatorname{diag}\left(\xi_{1}, \xi_{2}, \cdots, \xi_{\tilde{m}}\right), \quad \xi_{i}>0, i=1,2, \cdots, \tilde{m}, \\
\tilde{m} \triangleq \operatorname{rank}[H](\leq m) .
\end{gathered}
$$

Without loss of generality, we can assume that

$$
\xi_{1} \geq \xi_{2} \geq \cdots \geq \xi_{\tilde{m}}>0 \text {. }
$$

For any $\tilde{U} \in \boldsymbol{R}^{n \times \tilde{m}}$ with property $\tilde{U}^{\prime} \tilde{U}=I$ and for any $\tilde{\Xi}$ with properties (13) and (15), let

$$
\mathscr{H}(\tilde{U}, \tilde{\Xi}) \triangleq\left\{H \in \boldsymbol{R}^{m \times n} ; H^{\prime} R_{0}^{-1} H=\tilde{U} \tilde{\Xi} \tilde{U}^{\prime}\right\} .
$$

Thus, $\mathscr{H}(\tilde{U}, \tilde{\Xi})$ is the set of values of $H$ for which $H^{\prime} R_{0}^{-1} H$ has fixed values of the positive eigenvalues $\xi_{i}$ and the corresponding eigenvectors $u_{i}, i=1,2, \cdots, \tilde{m}$.

We have the following theorem for the solution $Q$ of (8) which guarantees that we can always take $H$ in the form (17) given below.

«Theorem 1» ${ }^{[18]}$ Assume $(\mathrm{C}-1)$ and $(\mathrm{C}-2)$. Then, we have the following properties. 
(i) $Q$, the solution of (8), is completely determined by $\left(A, G G^{\prime}, \tilde{U}, \tilde{\Xi}\right)$.

(ii) For a fixed set of values $(\tilde{U}, \tilde{\Xi})$ and for any $H \in \mathscr{H}(\tilde{U}, \tilde{\Xi})$, we have the same value of $Q$.

(iii) For any set of values $(\tilde{U}, \tilde{\Xi})$, an element in $\mathscr{H}(\tilde{U}, \tilde{\Xi})$ is given by

$$
H=R_{0}^{1 / 2} \tilde{\Gamma} \tilde{\Xi}^{1 / 2} \tilde{U}^{\prime}
$$

where $\tilde{\Gamma} \in \boldsymbol{R}^{m \times \tilde{m}}$ denotes the first $\tilde{m}$ columns of an orthogonal matrix $\Gamma \in \boldsymbol{R}^{m \times m}$ such that $\Gamma \Gamma^{\prime}=\Gamma^{\prime} \Gamma=I$.

(iv) One of the optimal values of $H \in \boldsymbol{R}^{m \times n}$ is given by the form (17).

Thus, without loss of generality, we can take $H$ as the form given by (17). Thus, the problem has been converted into the one of the optimization with respect to $\tilde{\Gamma} \in \boldsymbol{R}^{m \times \tilde{m}}$, $\tilde{U} \in \boldsymbol{R}^{n \times \tilde{m}}$ and $\tilde{\Xi} \triangleq \operatorname{diag}\left(\xi_{1}, \xi_{2}, \cdots, \xi_{\tilde{m}}\right)$.

[Remark 1] Theorem 1 implies that the optimal value of $H$ may not be unique but there are a number of minimum points in the space $\boldsymbol{R}^{m \times n}$ of $H$, and that by the change of variables $H \rightarrow(\tilde{U}, \tilde{\Xi})$, these minimum points are mapped into a single point in the space of $(\tilde{U}, \tilde{\Xi})$. This is a main reason that the method of optimization of $H$ given in this paper is efficient.

\section{The Condition of Optimality}

As we see from (8), (12) and (17), the value of $Q$ is independent of $\tilde{\Gamma} \in \boldsymbol{R}^{m \times \tilde{m}}$. Hence, the optimal value of $\tilde{\Gamma}$ should be determined in such a way that $\operatorname{tr}\left[H H^{\prime}\right]$, the second term in (11), is minimized. This minimization is easily done by the following theorem.

«Theorem 2»[18] Assume (C-1)-(C-2). Then the optimal value of $\tilde{\Gamma} \in \boldsymbol{R}^{m \times \tilde{m}}$ is given by the set of eigenvectors of $R_{0}$ corresponding to the first $\tilde{m}$ eigenvalues in ascending order, i.e., we have the relation

$$
\begin{gathered}
R_{0}=\left[\begin{array}{cc}
\tilde{\Gamma} & \bar{\Gamma}
\end{array}\right] \Psi\left[\begin{array}{l}
\tilde{\Gamma}^{\prime} \\
\bar{\Gamma}^{\prime}
\end{array}\right], \quad \Psi=\operatorname{diag}\left(\psi_{1}, \psi_{2}, \cdots, \psi_{\tilde{m}}, \cdots, \psi_{m}\right), \\
\psi_{1} \leq \psi_{2} \leq \cdots \leq \psi_{\tilde{m}} \leq \cdots \leq \psi_{m} .
\end{gathered}
$$

[Remark 2] If we take $\tilde{\Gamma} \in \boldsymbol{R}^{m \times \tilde{m}}$ according to Theorem 2, we have

$$
H=\tilde{\Gamma} \tilde{\Psi}^{1 / 2} \tilde{\Xi}^{1 / 2} \tilde{U}^{\prime},
$$

where

$$
\tilde{\Psi} \triangleq \operatorname{diag}\left(\psi_{1}, \psi_{2}, \cdots, \psi_{\tilde{m}}\right) .
$$

For the existence and the uniqueness of nonnegativedefinite and positive-definite solutions of (8), explicit conditions are known ([19; Sec. 13.5], [20]). Since we are assuming $G G^{\prime}>0$ by $(\mathrm{C}-1)$ and, therefore, $(A, G)$ is a controllable pair of matrices, we have the following theorem.

«Theorem 3» Assume (C-1) and (C-2). If $\left(\tilde{U}^{\prime}, A\right)$ is detectable and $\tilde{\Xi}$ satisfies $(15)$, then we have a unique positive-definite solution $Q$ of (8) with (12), and $A-Q \tilde{U} \tilde{\Xi} \tilde{U}^{\prime}$ is an asymptotically stable matrix.

(Proof) Combining (8) with (12), we have

$$
A Q+Q A^{\prime}+G G^{\prime}-Q \tilde{U} \tilde{\Xi} \tilde{U}^{\prime} Q=0 .
$$

From (21), we have the assertion by [19; Sec. 3.5], [20].

Along with the condition of Theorem 3, let us assume

(C-3) The set of $\tilde{U}$, determined by

$$
\mathscr{D}(A) \triangleq\left\{\tilde{U} \in \boldsymbol{R}^{n \times \tilde{m}} ; \tilde{U}^{\prime} \tilde{U}=I,\left(\tilde{U}^{\prime}, A\right) \text { is detectable }\right\},
$$

is not empty. Also, the optimal value of $\tilde{U}$ belongs to $\mathscr{D}(A)$.

By Theorem 2 and Theorem 3, Problem 1' is converted into the following form.

[Problem 2] Find $\tilde{U} \in \mathscr{D}(A)$ and $\tilde{\Xi}=\operatorname{diag}\left(\xi_{1}, \xi_{2}, \cdots\right.$, $\left.\xi_{\tilde{m}}\right)$ such that

$$
J=\operatorname{tr}[M Q]+\operatorname{tr}[\tilde{\Psi} \tilde{\Xi}] \rightarrow \min .
$$

subject to (21) and (15).

For Problem 2, the following result is already shown [18].

«Theorem 4 (Condition of Optimality)» Assume (C-1)(C-3). Let us define $F \in \boldsymbol{R}^{n \times n}$ by

$$
F \triangleq A-Q \tilde{U} \tilde{\Xi} \tilde{U}^{\prime} \text {. }
$$

Then, the condition of optimality of $(\tilde{U}, \tilde{\Xi})$ is given by

$$
Q X Q \tilde{U}=\tilde{U} \tilde{\Psi},
$$

where $X \in \boldsymbol{R}^{n \times n}$ is a solution of the Lyapunov equation:

$$
F^{\prime} X+X F+M=0 .
$$

Since $\tilde{\Psi}$ is a diagonal matrix given by (20), the relation given by (25) implies that

«Corollary 1» Assume that (C-1)-(C-3) are satisfied. The optimal $(\tilde{U}, \tilde{\Xi})$ is such that

(i) Each column vector of $\tilde{U}$ is an eigenvector of $Q X Q$ which is a symmetric matrix.

(ii) The order of column vectors in $\tilde{U}$ is the one that the corresponding eigenvalues are in ascending order.

(iii) The $\tilde{m}$ eigenvalues of $Q X Q$ that corresponds to $\tilde{U}$ coincide with the first $\tilde{m}$ eigenvalues of $R_{0}$.

[Remark 3] Since $M \in \boldsymbol{R}^{n \times n}$ is positive-definite and $F$ is asymptotically stable by Theorem 3, the Lyapunov equation (25) has a positive-definite symmetric solution $X \in \boldsymbol{R}^{n \times n}$. Hence, we have $Q X Q>0$.

\section{Solution of the Problem}

By Theorem 4, the solution of Problem 2 is given by a set of matrix equations for $(Q, \tilde{U}, \tilde{\Xi}, X)$ : 


$$
\begin{gathered}
A Q+Q A^{\prime}+G G^{\prime}-Q \tilde{U} \tilde{\Xi} \tilde{U}^{\prime} Q=0, \\
Q X Q \tilde{U}=\tilde{U} \tilde{\Psi}, \\
F^{\prime} X+X F+M=0, \\
F \triangleq A-Q \tilde{U} \tilde{\Xi} \tilde{U}^{\prime},
\end{gathered}
$$

with the constraints:

$$
\tilde{U} \in \mathscr{D}(A),
$$

and

$$
\xi_{1} \geq \xi_{2} \geq \cdots \geq \xi_{\tilde{m}}>0
$$

Clearly, the optimal observation gain matrix is given by (19) if we obtain the solution $(Q, \tilde{U}, \tilde{\Xi}, X)$ of the above set of equations. In this section, a method of solving this set of equations will be developed. In [18], considering the case of $\tilde{m}=m=n$, both for $\tilde{\Psi}=I$ and $\tilde{\Psi} \neq I$, simple algorithms are proposed and available. The performances of these algorithms are very good although mathematical proofs of the convergency are not provided yet. However, the same kind of algorithm is, at present, not applicable for more general case $\tilde{m} \leq m<n$. Here, we develop an algorithm which can be also applied to the case $\tilde{m} \leq m<n$ in addition to the above cases.

First, let us note that for $W \in \boldsymbol{R}^{n \times n}$ defined by

$$
W \triangleq Q X Q,
$$

we have the following equation from (25) and (21) ${ }^{[18]}$.

«Theorem 5» Assume (C-1)-(C-3). $W \triangleq Q X Q$ satisfies

$$
\left(A+G G^{\prime} Q^{-1}\right) W+W\left(A+G G^{\prime} Q^{-1}\right)^{\prime}-Q M Q=0,
$$

and

$$
W=\tilde{U} \tilde{\Psi} \tilde{U}^{\prime}+\left(I-\tilde{U} \tilde{U}^{\prime}\right) W\left(I-\tilde{U} \tilde{U}^{\prime}\right) .
$$

Furthermore, $-\left(A+G G^{\prime} Q^{-1}\right)$ is asymptotically stable.

(Proof) See [18; Theorem 5].

Note the facts that when $(\tilde{U}, \tilde{\Xi})$ is given, (21) is a matrix Riccati equation for $Q$ and that when $Q$ is given, (28) is a Lyapunov equation for $W$. Hence, let us denote

$$
\begin{aligned}
& \mathscr{Q}_{L}(A, G) \triangleq\left\{Q \in \boldsymbol{R}^{n \times n} ; Q^{\prime}=Q, Q>0,\right. \\
& \left.-\left(A+G G^{\prime} Q^{-1}\right) \text { is assymptotically stable }\right\} \text {, } \\
& \mathscr{Q}_{R}(A, G) \triangleq\left\{Q \in \boldsymbol{R}^{n \times n} ; Q\right. \text { is a solution of (21) } \\
& \text { with } \tilde{U} \in \mathscr{D}(A), \tilde{\Xi}>0\} \text {, }
\end{aligned}
$$

Then, we see that

«Theorem 6» Assume (C-1)-(C-3).

(i) For any $(\tilde{U}, \tilde{\Xi}), \tilde{U} \in \mathscr{D}(A), \tilde{\Xi}>0$, we have a unique solution $Q \in \mathscr{Q}_{R}(A, G)$ of (21), which is not optimal in general, and which is obtained by the equilibrium solution of

$$
\frac{d Q_{t}}{d t}=A Q_{t}+Q_{t} A^{\prime}+G G^{\prime}-Q_{t} \tilde{U} \tilde{\Xi} \tilde{U}^{\prime} Q_{t} .
$$

(ii) For any $Q \in \mathscr{Q}_{L}(A, G)$, we have a unique solution $W \in \mathscr{V}_{L}(A, G, M)$ of (28), which is not optimal in general, and which is obtained by the equilibrium solution of

$\frac{d W_{s}}{d s}=-\left(A+G G^{\prime} Q^{-1}\right) W_{s}-W_{s}\left(A+G G^{\prime} Q^{-1}\right)^{\prime}+Q M Q$.

(iii) $\mathscr{Q}_{R}(A, G) \subset \mathscr{Q}_{L}(A, G)$ and $\mathscr{V}_{R}(A, G, M) \subset \mathscr{\mathscr { V }} L(A, G, M)$.

萳Proof) The statements (i)-(iii) are clear by the definitions of $\mathscr{Q}_{L}(A, G), \mathscr{Q}_{R}(A, G), \mathscr{V}_{L}(A, G, M) \quad$ and $\mathscr{W}_{R}(A, G, M)$.

Thus, for any $(\tilde{U}, \tilde{\Xi}), \tilde{U} \in \mathscr{D}(A), \tilde{\Xi}>0$, we have a unique solution $Q \in \mathscr{Q}_{R}(A, G)$ of (21), and then, we have a unique solution $W \in \mathscr{W}_{R}(A, G, M)$ of (28) for $Q \in \mathscr{Q}_{R}(A, G)$. Namely, for any $(\tilde{U}, \tilde{\Xi}), \tilde{U} \in \mathscr{D}(A), \tilde{\Xi}>0$, we have a unique solution $(Q, W), Q \in \mathscr{Q}_{R}(A, G), W \in \mathscr{V}_{R}(A, G, M)$ of the set of equations (21) and (28). Hence, we have the solution of the optimization problem if we find a solution $(Q, W)$ of (21) and (28) which satisfies (29) or (24).

Now, let us consider the following differential equation which is obtained by replacing $(\tilde{U}, \tilde{\Xi})$ in (34) with $\left(\tilde{U}_{k}, \tilde{\Xi}_{k}\right), \quad k=0,1,2, \cdots$.

$$
\frac{d Q_{t}^{k}}{d t}=A Q_{t}^{k}+Q_{t}^{k} A^{\prime}+G G^{\prime}-Q_{t}^{k} \tilde{U}_{k} \tilde{\Xi}_{k} \tilde{U}_{k}^{\prime} Q_{t}^{k} .
$$

Here, we assume that $\left(\tilde{U}_{k}, \tilde{\Xi}_{k}\right)$ satisfies the same properties as (C-3) and (15). Then, we have the equilibrium solution $Q_{k}$ of (36) for any proper initial value $Q_{0}^{k}$. By replacing $Q$ in (35) with $Q_{k}$, we can compute the equilibrium solution $W_{k}$ of

$$
\frac{d W_{s}^{k}}{d s}=-\left(A+G G^{\prime} Q_{k}^{-1}\right) W_{s}^{k}-W_{s}^{k}\left(A+G G^{\prime} Q_{k}^{-1}\right)^{\prime}+Q_{k} M Q_{k} .
$$

Thus, we notice the following fact.

«Theorem 7» Assume (C-1)-(C-3). For any $\left(\tilde{U}_{k}, \tilde{\Xi}_{k}\right)$, $\tilde{U}_{k} \in \mathscr{D}(A), \quad \tilde{\Xi}_{k}=\operatorname{diag}\left(\tilde{\xi}_{1}^{k}, \tilde{\xi}_{1}^{k}, \cdots, \tilde{\xi}_{\tilde{m}}^{k}\right), \quad \tilde{\xi}_{1}^{k} \geq \tilde{\xi}_{2}^{k} \geq \cdots \geq \tilde{\xi}_{\tilde{m}}^{k}>0$, and for the equilibrium solution $\left(Q_{k}, W_{k}\right)$ of (36) and (37), the set of values: $(Q, \tilde{U}, \tilde{\Xi}, W)=\left(Q_{k}, \tilde{U}_{k}, \tilde{\Xi}_{k}, W_{k}\right)$ satisfies both (21) and (28).

Of course, in general, the set of eigenvectors $\hat{U}_{k}$ of $W_{k}$ does not coincide with $\tilde{U}_{k}$, and the set of eigenvalues $\hat{\Psi}_{k}$ of $W_{k}$ does not equal to $\tilde{\Psi}$ either, i.e., (29) does not hold for $(\tilde{U}, W)=\left(\tilde{U}_{k}, W_{k}\right)$, and $\left(\tilde{U}_{k}, \tilde{\Xi}_{k}\right)$ is not optimal. 
As we can see, if $\left(\tilde{U}_{k}, \tilde{\Xi}_{k}\right)$ is updated, by using the equilibrium solution $\left(Q_{k}, W_{k}\right)$ of (36) and (37), in such a way that $\left(\tilde{U}_{k}, \tilde{\Xi}_{k}\right) \rightarrow(\tilde{U}, \tilde{\Xi})$ as $k \rightarrow \infty$, then we have the value of $(Q, \tilde{U}, \tilde{\Xi}, W)$ for which we have (29) in addition to (21) and (28). If this is possible, we have the solution of Problem 2.

[Remark 4] Note that this transversal update method also works for the cases when one of the values of $\left(\tilde{U}_{k}, \tilde{\Xi}_{k}\right)$ is fixed and constant.

(iv) When $\quad \tilde{\Xi}_{k} \equiv \tilde{\Xi}_{0}$, where $\quad \tilde{\Xi}_{0}$ is a positive-definite diagonal matrix, compute the equilibrium solution $\left(Q_{k}, W_{k}\right)$ of (37) and

$$
\frac{d Q_{t}^{k}}{d t}=A Q_{t}^{k}+Q_{t}^{k} A^{\prime}+G G^{\prime}-Q_{t}^{k} \tilde{U}_{k} \tilde{\Xi}_{0} \tilde{U}_{k}^{\prime} Q_{t}^{k} .
$$

With proper updates of $\tilde{U}_{k}$ such that $\tilde{U}_{k} \rightarrow \tilde{U}^{*}$ as $k \rightarrow \infty$, we have the value $(Q, \tilde{U}, W)=\left(Q_{k}, \tilde{U}_{k}, W_{k}\right)$ for which we have $\tilde{U}^{*}=\tilde{U}_{k}=\hat{U}_{k}$ in addition to (21) and (28) with $\tilde{\Xi}_{k} \equiv \tilde{\Xi}_{0}$. It was shown in [18], that for any value $\tilde{\Xi}_{0}$, we have $\tilde{U}^{*}$ which is very close to the optimal value $\tilde{U}$.

(v) When $\tilde{U}_{k} \equiv \tilde{U}_{0}$, where $\tilde{U}_{0}$ satisfies $\tilde{U}_{0}^{\prime} \tilde{U}_{0}=I$, compute the equilibrium solution $\left(Q_{k}, W_{k}\right)$ of (37) and

$$
\frac{d Q_{t}^{k}}{d t}=A Q_{t}^{k}+Q_{t}^{k} A^{\prime}+G G^{\prime}-Q_{t}^{k} \tilde{U}_{0} \tilde{\Xi}_{k} \tilde{U}_{0}^{\prime} Q_{t}^{k} .
$$

With proper updates of $\tilde{\Xi}_{k}$ such that $\tilde{\Xi}_{k} \rightarrow \tilde{\Xi}^{*}$ as $k \rightarrow \infty$, we have the value $(Q, \tilde{\Xi}, W)=\left(Q_{k}, \tilde{\Xi}_{k}, W_{k}\right)$ for which we have $\hat{\Psi}_{k}=\tilde{\Psi}$ in addition to (21) and (28) with $\tilde{U} \equiv \tilde{U}_{0}$.

In fact, the alternate search by the separate update (i) and (ii) works fine because in (i), we can usually get a value $\tilde{U}^{*}$ which is very close to the optimal value $\tilde{U}$.

\section{Numerical Algorithms}

In this section, an algorithm is proposed for Problem 2 based on the discussions in the previous section. We employ the following simple update methods of $\tilde{U}_{k}$ and $\tilde{\Xi}_{k}$.

With the equilibrium solution $Q_{k}$ of (36) and the initial value given by

$$
W_{0}^{k} \triangleq \tilde{U}_{k} \tilde{\Psi} \tilde{U}_{k}^{\prime}+\left(I-\tilde{U}_{k} \tilde{U}_{k}^{\prime}\right) W_{k-1}\left(I-\tilde{U}_{k} \tilde{U}_{k}^{\prime}\right),
$$

let $W_{k}$ be the equilibrium solution of (37). Then, define $\hat{U}_{k}$ and $\hat{\Psi}_{k}$ by the relation:

$$
W_{k}=\hat{U}_{k} \hat{\Psi}_{k} \hat{U}_{k}^{\prime}+\left(I-\hat{U}_{k} \hat{U}_{k}^{\prime}\right) W_{k}\left(I-\hat{U}_{k} \hat{U}_{k}^{\prime}\right) \text {. }
$$

Namely, $\hat{U}_{k} \in \boldsymbol{R}^{\tilde{m}}$ is the set of eigenvectors of $W_{k}$ with property $\hat{U}_{k}^{\prime} \hat{U}_{k}=I$ and $\hat{\Psi}_{k}$ is the corresponding set of eigenvalues in ascending order:

$$
\begin{gathered}
\hat{\Psi}_{k}=\operatorname{diag}\left(\hat{\Psi}_{1}^{k}, \hat{\psi}_{1}^{k}, \cdots, \hat{\Psi}_{\tilde{m}}^{k}\right), \\
0<\hat{\Psi}_{1}^{k} \leq \hat{\psi}_{2}^{k} \leq \cdots \leq \hat{\Psi}_{\tilde{m}}^{k} .
\end{gathered}
$$

Thus, for the condition of optimality (29) to be satisfied, we must have both $\tilde{U}_{k}=\hat{U}_{k}$ and $\hat{\Psi}_{k}=\tilde{\Psi}$.

(I) Let

$$
\begin{aligned}
\hat{Z}_{k} & \triangleq(1+\alpha) \tilde{U}_{k} \tilde{\Xi}_{k} \tilde{U}_{k}^{\prime}-\alpha \hat{U}_{k} \tilde{\Xi}_{k} \hat{U}_{k}^{\prime} \\
& =\tilde{U}_{k} \tilde{\Xi}_{k} \tilde{U}_{k}^{\prime}-\alpha\left(\hat{U}_{k} \tilde{\Xi}_{k} \hat{U}_{k}^{\prime}-\tilde{U}_{k} \tilde{\Xi}_{k} \tilde{U}_{k}^{\prime}\right),
\end{aligned}
$$

where $0 \leq \alpha<1$. Then, the updated value $\tilde{U}_{k+1}$ is determined by the $\tilde{m}$ eigenvectors of $\hat{Z}_{k}$ corresponding to the first $\tilde{m}$ eigenvalues in descending order, namely, by the relation

$$
\hat{Z}_{k}=\tilde{U}_{k+1} \hat{\Xi}_{k} \tilde{U}_{k+1}^{\prime}+\delta, \quad \delta \cong 0,
$$

where $\hat{\Xi}_{k}=\operatorname{diag}\left(\hat{\xi}_{1}^{k}, \hat{\xi}_{1}^{k}, \cdots, \hat{\xi}_{\tilde{m}}^{k}\right)$ and $\hat{\xi}_{1}^{k} \geq \hat{\xi}_{2}^{k} \geq \cdots \geq \hat{\xi}_{\tilde{m}}^{k}>0$.

(II) The updated value $\tilde{\Xi}_{k+1}$ is determined, using the eigenvalue $\hat{\psi}_{i}, \quad i=1,2, \cdots, \tilde{m}$ of $W_{k}$, by the relation:

$$
\begin{aligned}
\tilde{\Xi}_{k+1} & =\tilde{\Xi}_{k}\left\{(1-\beta) I+\beta \hat{\Psi}_{k} \tilde{\Psi}^{-1}\right\} \\
& =\tilde{\Xi}_{k}\left\{I+\beta\left(\hat{\Psi}_{k} \tilde{\Psi}^{-1}-I\right)\right\},
\end{aligned}
$$

where $0 \leq \beta<1$.

[Remark 5] It should be noted that the new value $\tilde{U}_{k+1}$ in (45) is determined by the exterior division (44) with $\tilde{U}_{k}$ and $\hat{U}_{k}$ on the old value side, i.e., $\tilde{U}_{k}$-side. This is seen to be correct for all the cases that we have checked.

[Remark 6] It should be noted that the above updates of $\tilde{U}_{k}$ and $\tilde{\Xi}_{k}$ are separately applicable. Especially, for the case $\tilde{m} \leq m<n$, it is rather difficult to get the correct ordering of the eigenvalues and eigenvectors of $W_{k}$ at the early stage of computation. This difficulty is avoided by the multi-stage computations in the following way.

(i) Fixing $\tilde{\Xi}_{k} \equiv \tilde{\Xi}_{0}$ and updating $\tilde{U}_{k}$ by (44) and (45), find $\tilde{U}^{*}$ such that we have $\tilde{U}_{k}=\hat{U}_{k}=\tilde{U}^{*}$ for (41), and that both (21) and (28) hold for $(Q, \tilde{U}, \tilde{\Xi}, W)=$ $\left(Q_{k}, \tilde{U}^{*}, \tilde{\Xi}_{0}, W_{k}\right)$.

(ii) Fixing $\tilde{U}_{k} \equiv \tilde{U}^{*}$ and updating $\tilde{\Xi}_{k}$ by (46), find $\tilde{\Xi}_{k}$ such that for (41), we have

$$
\hat{\Psi}_{k}=\tilde{\Psi},
$$

and that both (21) and (28) hold for $(Q, \tilde{U}, \tilde{\Xi}, W)=$ $\left(Q_{k}, \tilde{U}^{*}, \tilde{\Xi}_{k}, W_{k}\right)$.

(iii) Updating both $\tilde{U}_{k}$ and $\tilde{\Xi}_{k}$ by (44)-(46), find $\left(\tilde{U}_{k}, \tilde{\Xi}_{k}\right)$ such that we have both $\tilde{U}_{k}=\hat{U}_{k}$ and $\hat{\Psi}_{k}=\tilde{\Psi}$ for (41), and that both (21) and (28) hold for $(Q, \tilde{U}, \tilde{\Xi}, W)=\left(Q_{k}, \tilde{U}_{k}, \tilde{\Xi}_{k}, W_{k}\right)$.

\section{Numerical Examples}

In this section, we will show the results of numerical 
calculations for a simple 3-dimensional system.

Example $1(n=m=\tilde{m}=3)$. Let us consider a 3-dimensional system with

$$
\begin{gathered}
A=\left[\begin{array}{rrr}
-0.10 & -0.03 & 0.02 \\
0.02 & -0.10 & 0.01 \\
0.06 & 0.03 & -0.08
\end{array}\right], \quad G=\left[\begin{array}{ccc}
0.5 & 0 & 0 \\
0 & 0.5 & 0 \\
0 & 0 & 0.5
\end{array}\right], \\
M=\left[\begin{array}{lll}
28.920 & 33.528 & 18.723 \\
33.528 & 66.742 & 51.376 \\
18.723 & 51.376 & 48.180
\end{array}\right] .
\end{gathered}
$$

The numerical computations of the proposed algorithm have been carried out for the following three cases.

(Case 1: $n=m=\tilde{m}=3$ ). Let $m=\tilde{m}=3$ and

$$
\begin{aligned}
R_{0} & =\left[\begin{array}{ccc}
-\frac{1}{8} & -\frac{3 \sqrt{3}}{8} & \frac{3}{4} \\
-\frac{3 \sqrt{3}}{8} & \frac{5}{8} & \frac{\sqrt{3}}{4} \\
-\frac{3}{4} & -\frac{\sqrt{3}}{4} & -\frac{1}{2}
\end{array}\right]\left[\begin{array}{ccc}
0.49 & 0 & 0 \\
0 & 1.0 & 0 \\
0 & 0 & 1.44
\end{array}\right]\left[\begin{array}{ccc}
-\frac{1}{8} & -\frac{3 \sqrt{3}}{8} & -\frac{3}{4} \\
-\frac{3 \sqrt{3}}{8} & \frac{5}{8} & -\frac{\sqrt{3}}{4} \\
\frac{3}{4} & \frac{\sqrt{3}}{4} & -\frac{1}{2}
\end{array}\right] \\
& =\left[\begin{array}{rrr}
1.2395 & 0.1015 & -0.2128 \\
0.1015 & 0.8673 & -0.3437 \\
-0.2128 & -0.3437 & 0.8231
\end{array}\right],
\end{aligned}
$$

i.e.,

$$
\tilde{\Psi}=\Psi=\operatorname{diag}(0.49,1.0,1.44), \quad \tilde{\Gamma}=\Gamma=\left[\begin{array}{ccc}
-\frac{1}{8} & -\frac{3 \sqrt{3}}{8} & \frac{3}{4} \\
-\frac{3 \sqrt{3}}{8} & \frac{5}{8} & \frac{\sqrt{3}}{4} \\
-\frac{3}{4} & -\frac{\sqrt{3}}{4} & -\frac{1}{2}
\end{array}\right] \text {. }
$$

(Case 2: $n=3, m=\tilde{m}=2$ ). Let $m=\tilde{m}=2$ and

$$
\begin{aligned}
R_{0} & =\left[\begin{array}{cc}
\frac{1}{\sqrt{5}} & \frac{2}{\sqrt{5}} \\
-\frac{2}{\sqrt{5}} & \frac{1}{\sqrt{5}}
\end{array}\right]\left[\begin{array}{cc}
0.49 & 0 \\
0 & 1.0
\end{array}\right]\left[\begin{array}{cc}
\frac{1}{\sqrt{5}} & -\frac{2}{\sqrt{5}} \\
\frac{2}{\sqrt{5}} & \frac{1}{\sqrt{5}}
\end{array}\right] \\
& =\left[\begin{array}{ll}
0.89800 & 0.20400 \\
0.20400 & 0.59200
\end{array}\right],
\end{aligned}
$$

i.e.,

$$
\tilde{\Psi}=\Psi=\operatorname{diag}(0.49,1.0), \quad \tilde{\Gamma}=\Gamma=\left[\begin{array}{cc}
\frac{1}{\sqrt{5}} & \frac{2}{\sqrt{5}} \\
-\frac{2}{\sqrt{5}} & \frac{1}{\sqrt{5}}
\end{array}\right] .
$$

(Case 3: $n=3, m=\tilde{m}=1$ ). $\quad$ Let $m=\tilde{m}=1$ and

$$
R_{0}=0.49 \text {, }
$$

i.e.,

$$
\tilde{\Psi}=\Psi=\psi_{1}=0.49, \quad \Gamma=1 .
$$

The result of the computation for all these three cases are summarized in Table 1. It is seen from Table 1 that for three cases, only small changes are seen in the values of $\tilde{U}$ and $\tilde{\Xi}$ although, in other variables, especially in $Q$ and $W$, we see rather big changes in their values. This is the fact should be noted.

Table 1. The result by the proposed algorithm (Case 1, Case2 and Case 3)

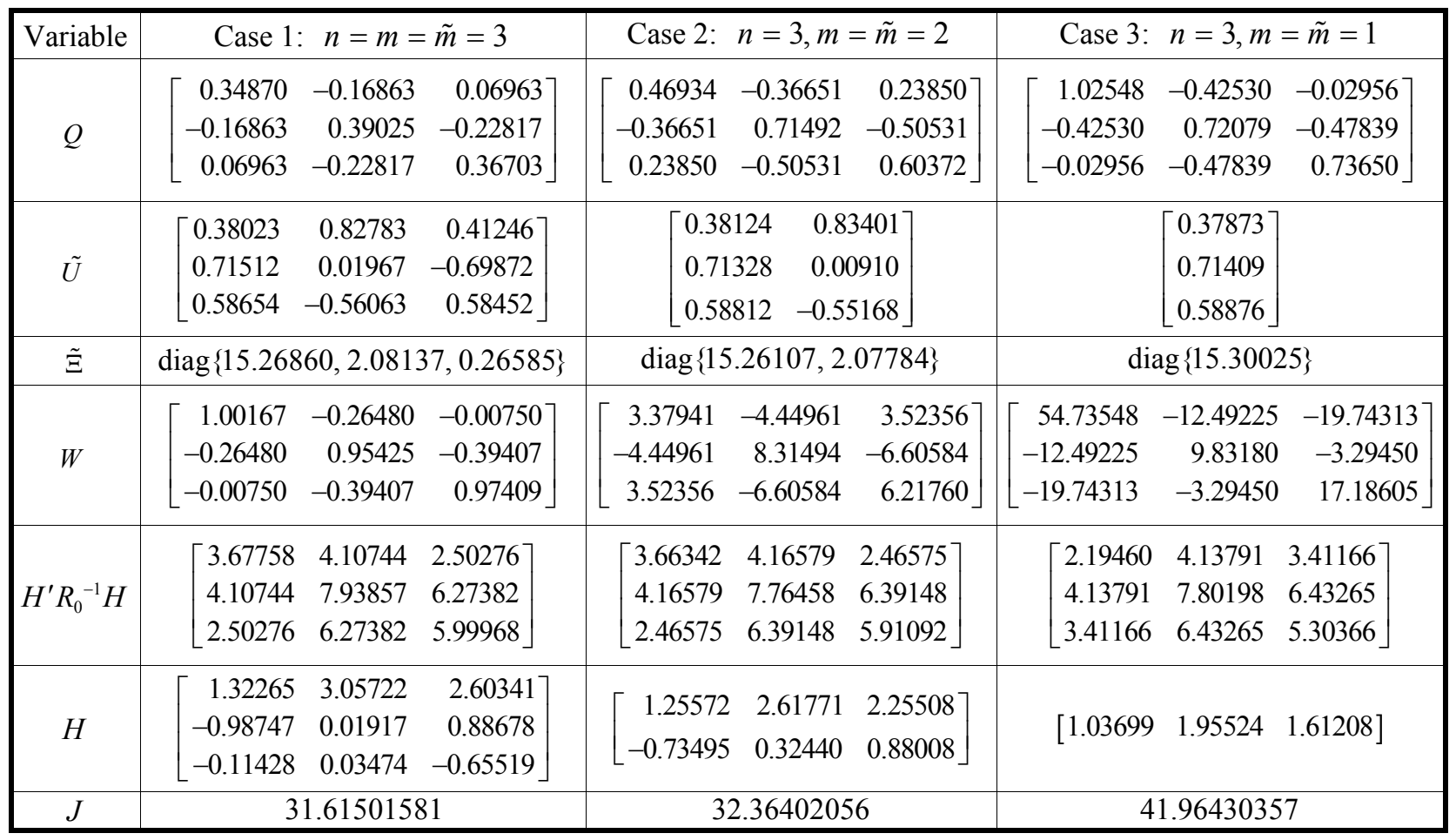




\section{Conclusions}

In this paper, we considered the optimization of the observation gain matrix for the stationary Kalman-Bucy filter. The optimal gain matrix is obtained from the eigenvalues and the eigenvectors of SSNRM which is a symmetric matrix of a quadratic function of the gain matrix and is linearly dependent on the inverse of the observation noise covariance matrix.

In this paper, a recursive algorithm was proposed to compute the optimal value of SSNRM by solving the Riccati and Lyapunov equations alternately and by updating the values of eigenvalues and eigenvectors of SSNRM according to the condition of optimality.

At present, no mathematical proof of the convergence of the proposed algorithm is provided yet. However, as far as the numerical experiments are concerned, the performance of the algorithm is good. At least, it is easy for us to get nearly optimal value of the observation gain by the proposed algorithm.

The solutions of algebraic Riccati and Lyapunov equations were, in this paper, computed as the equilibrium solutions of the corresponding differential equations. However, it is clear that any other methods to compute the solutions of these equations are also applicable in the proposed algorithm.

\section{References}

[1] M. Athans, "On the determination of optimal costly measurement strategies for linear stochastic systems," Prep. 5th IFAC World Congress, Paris, 1/11, 1972.

[2] A. Logothetis, A. Isaksson and E. Skadas, "On sensor scheduling via information theoretic criteria," Prep. ACC, 1999.

[3] M. Aoki, and M. T. Li, "Optimal discrete time control system with cost for observation," IEEE Trans. Automatic Control, AC-14, 2, 165/175, 1969.

[4] A. Sano and M. Terao, "Measurement optimization in optimal process control," Automatica, 5, 705/714, 1970.

[5] S. Ihara, "Coding theory in white Gaussian channel with feedback," J. Multi-variate Analysis, 4, 74/87, 1974.

[6] Y. Takeuchi, "The optimal transmission of non-Gaussian signals through a noisy channel with feedback," IEEE Trans. Inform. Theory, IT-40, 5, 1624/1629, 1994.

[7] Y. Takeuchi and A. Ida, "Optimal Transmission of a set of Gaussian Signals through Discrete-Time Channels with Feedback," Proc. of 35th ISCIE SSS, Ube, Oct. 30-31, 2003, 118/123, 2004.
[8] Y. Takeuchi, "An information theoretic approach to optimization of linear observations for the Kalman-Bucy filter," International Journal of Innovative Computing, Information \& Control, 1-3, 401/416, 2005.

[9] Y. Takeuchi, "Optimal transmission of a set of discrete-time Gaussian signals through channels with feedback," International Journal of Innovative Computing, Information \& Control, 2-5, 927/942, 2006.

[10] Y. Takeuchi, "Optimization of linear observations for the stationary Kalman filter based on a generalized Water Filling Theorem," International Journal of Innovative Computing, Information \& Control, 4-1, 211/230, 2008.

[11] Y. Takeuchi and A. Hirata, "A method of optimization of linear observations for the Kalman filter based on a generalized Water Filling Theorem," International Journal of Innovative Computing, Information \& Control, 5-1, 75/85, 2009.

[12]Y. Takeuchi and M Inoue, "Optimization of observations for LQG control systems by an information theoretic approach," International Journal of Innovative Computing, Information \& Control, 6-1, 75/87, 2010.

[13] Y. Takeuchi, "Optimization of linear observations for the stationary Kalman filter under a quadratic performance criterion," International Journal of Innovative Computing, Information \& Control, 7-1, 85/99, 2011.

[14] Y. Takeuchi, "Optimization of the observations for stationary LQG stochastic control systems under a quadratic criterion," International Journal of Innovative Computing, Information \& Control, 8-3, 2313/2328, 2012.

[15] Y. Takeuchi and R. Matsumi, "Hybrid optimization of the observations for stationary LQG control systems," Trans. ISCIE, 25-12, 366/374, 2012.

[16] Y. Takeuchi and H. Nakai, "A New Method of Optimization of Linear Observations for the Stationary Kalman Filter," Proc. of 44th ISCIE SSS, Tokyo, Nov. 1-2, 2012, 21/28, 2013.

[17] Y. Takeuchi and H. Nakai, "An Improved Method of Optimization of Linear Observations for the Stationary Kalman Filter," Proc. of 45th ISCIE SSS, Okinawa, Nov. 1-2, 2013, 1/8, 2014.

[18] Y. Takeuchi, "Optimization of Linear Observations for the Stationary Kalman-Bucy Filter," Trans. ISCIE, 28-12, 467/475, 2015.

[19]S. Kodama, and N. Suda, Matrix Theory for Systems Control (in Japanese), SICE, 1978.

[20] V. Kučera, "A contribution to matrix quadratic equations," IEEE Trans. Automatic Control, AC-17, 344/347, 1972. 